\title{
Topological Bose-Mott Insulators in a One-Dimensional Optical Superlattice
}

\author{
Shi-Liang Zhu, ${ }^{1,2,3}$ Z. D. Wang, ${ }^{3}$ Y.-H. Chan, ${ }^{4,5}$ and L. -M. Duan ${ }^{4,5}$ \\ ${ }^{1}$ National Laboratory of Solid State Microstructures, \\ Department of Physics, Nanjing University, Nanjing, China \\ ${ }^{2}$ Laboratory of Quantum Information Technology and SPTE, \\ South China Normal University,Guangzhou, China \\ ${ }^{3}$ Department of Physics and Center of Theoretical and Computational Physics, \\ The University of Hong Kong,Pokfulam Road, Hong Kong, China \\ ${ }^{4}$ Department of Physics, University of Michigan, Ann Arbor, Michigan 48109, USA \\ ${ }^{5}$ Center for Quantum Information, IIIS, Tsinghua University, Beijing, China
}

\begin{abstract}
We study topological properties of the Bose-Hubbard model with repulsive interactions in a onedimensional optical superlattice. We find that the Mott insulator states of the single-component (two-component) Bose-Hubbard model under fractional fillings are topological insulators characterized by a nonzero charge (or spin) Chern number with nontrivial edge states. For ultracold atomic experiments, we show that the topological Chern number can be detected through measuring the density profiles of the bosonic atoms in a harmonic trap.
\end{abstract}

PACS numbers: 03.75.Lm, 05.30.Rt, 73.21.Cd

Introduction - Ultracold atoms in optical lattices can be used to simulate strongly correlated many-body models that are central to the understanding of condensed matter physics. This simulation has attracted a lot of attention as the optical lattice experiments offer unparalleled controllability and new tools to study manybody physics 1- 4]. As a remarkable example, the BoseHubbard (BH) model has been experimentally realized with ultracold atoms and a quantum phase transition from a superfluid to a Mott insulator described by this model has been observed [3]. On the other hand, topological matters, such as quantum Hall systems and topological insulators, are of fundamental importance in physics [5]. Recently, studying topological phases with ultracold atoms has raised great interest [4, 6 - 12]. In general, it requires complicated control of experimental systems to realize topological phases with ultracold atoms. An interesting question is whether one can observe topological phases in a simple BH type of model, which can be readily implemented by many experimental groups. Topological properties of bosonic systems, however, have not been well-studied in literature, partly for the reason that the topological invariants are usually defined as an integration over all the occupied states in the momentum space [13, 14]. This definition does not apply directly to the bosonic system as many bosons can occupy the same momentum state.

In this Letter, in contrast to the conventional wisdom, we show that the BH model in a one-dimensional (1D) optical superlattice displays nontrivial topological properties. We demonstrate that the Mott insulators of the single-component (two-component) $\mathrm{BH}$ model at fractional fillings belong to topological matter with its phase characterized by a nonzero integer charge (or spin) Chern number. For Mott insulators, the bulk system has a gap in the excitation spectrum induced by the interaction.
For a topologically nontrivial Mott insulator state characterized by a nonzero Chern number, we further show that there are protected edge states inside the bulk gap under the open boundary condition. The Mott insulators at integer fillings for this system remain topologically trivial with a zero Chern number and no edge states. The topological properties discussed here are reminiscent of those in topological Mott insulators theoretically predicted in Ref. 15] for the Fermi-Hubbard model in a honeycomb lattice with frustrated next-neighbor interactions. Remarkably, we here show that topological Mott insulators can appear in a simple 1D BH model in an optical superlattice, which, besides being conceptually interesting, makes the experimental realization of topological matter much easier in the ultracold atomic system. We propose a scheme to detect the topological Chern number by observation of the plateaus of the density profile with ultracold atoms in a weak global harmonic trap as it is the case for experiments.

Single-component BH model in a superlattice.- We consider a single-component bosonic gas loaded into a $1 \mathrm{D}$ optical lattice, which is described by the $\mathrm{BH}$ model

$$
H=-J \sum_{\langle i j\rangle} b_{j}^{\dagger} b_{i}+\sum_{j}\left[U n_{j}\left(n_{j}-1\right) / 2+V_{j} n_{j}\right]
$$

where $V_{j}=V \cos (2 \pi \alpha j+\delta)$ denotes a periodic superlattice potential [16], $b_{j}$ and $b_{j}^{\dagger}$ correspond to the bosonic annihilation and creation operators of atoms on the $j$ th lattice site, $n_{j}=b_{j}^{\dagger} b_{j}$ is the number operator, and $J$ and $U$ represent the hopping rate and the on site interaction strength, respectively. We consider in this Letter a commensurate superlattice potential $V_{j}$ with $\alpha=p / q(p, q$ are integers) being a rational number and $\delta$ an arbitrary tunable phase, which has been experimentally realized [16]. We take $J$ as the energy unit by setting $J=1$.

The ground-state phase diagram of the Hamiltonian 
[Eq.(1)] is well-studied [2, 3, 17]. For a sufficiently large $U$, the system is in a gapped Mott insulator phase at commensurate fillings with $\nu \equiv N_{b} / N=m \alpha$, where $m$ is an integer, $N_{b}$ is the atom number, and $N$ is the number of lattice sites. Away from the commensurate fillings or for a small $U$, the system is in a superfluid state [17]. In this Letter, we focus on study of the topological properties of the Mott insulator phase.

The energy gap and the Chern number of the ground state.- The topological property is best characterized by the Chern number. To calculate the Chern number, we first perform exact diagonalization of the Hamiltonian [Eq.(1)] on a chain of $N$ sites with periodic or open boundary conditions [18]. The ground state is nondegenerate and separated from the higher eigenstates by a finite gap $\Delta$ at the commensurate fillings. This gap is shown in Fig.1 as a function of the interaction strength $U$ at $\nu=1 / 3$. The gap increases monotonically with $U$ and then saturates at a finite value. For a large $U$, the atoms become hard-core bosons. In this case, each site is occupied by no more than one atom. The hard-core boson Hubbard model can be mapped to a model of free fermions. From that mapping, we find that the saturation value of the gap is 1.08 (in units of $J$ ) at a large $U$ for an infinite system. The gap should decrease to zero as $U$ drops below a critical value $U_{c}$ where the system transits to a superfluid phase. In Fig. 1, due to the finite size effect, the saturation value of the energy gap is above 1.08 for the large $U$ and does not drop exactly to zero as $U$ diminishes. However, as the number of lattice sites increases, we clearly see the tendency that the gap approaches these limiting values at the two ends.

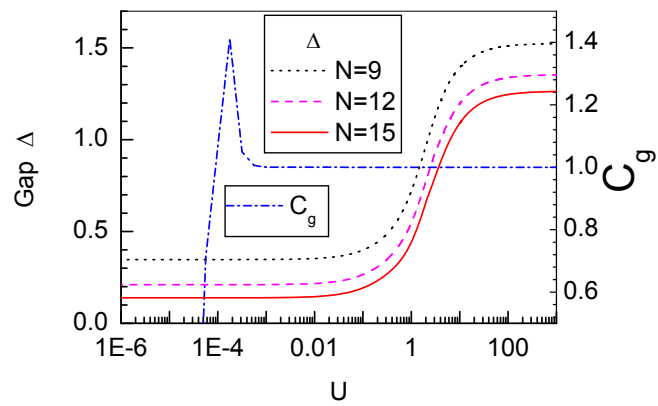

FIG. 1: (Color online) The energy gap $\Delta$ and the Chern number $C_{g}$ [ defined by Eq.(2)] as functions of the interaction strength $U$. The number of lattice sites $N=9,12,15$ are used for exact diagonalization, and we take $N=15$ for calculation of $C_{g}$. Other parameters include $V=1.5, \delta=2 \pi / 3$, $\alpha=1 / 3$, and $\nu=1 / 3$.

Now, we investigate the topological property of the system by calculating the Chern number. For fermions, the Chern number is defined as an integration over the occupied states in the momentum space [13]. This definition can not be extended to the bosonic system as many bosons can occupy the same momentum state. Fortu- nately, there is another way to calculate the Chern number for interacting systems [19]: suppose the ground state has a gap to the excited state and it depends on the parameters $\theta, \delta$ through a generalized periodic boundary condition $|\Psi(j+N, \theta, \delta)\rangle=e^{i \theta}|\Psi(j, \theta, \delta)\rangle$, where $j$ denotes an arbitrary site, $\theta$ is the twist angle, and $\delta$ is the phase in the superlattice potential $V_{j}$. Under this boundary condition, we numerically diagonalize Hamiltonian [Eq.(11)] and derive the ground state $|\Psi(\theta, \delta)\rangle$, which is a non-degenerate state separated from the excited state by a nonzero energy gap $\Delta$ when $U>U_{c}$. For the ground state $|\Psi(\theta, \delta)\rangle$ where $\theta$ and $\delta$ vary on a torus, one can define the Chern number $C_{g}$ as a topological invariant by the following formula [19]

$$
C_{g}=\frac{1}{2 \pi} \int_{0}^{2 \pi} d \theta \int_{0}^{2 \pi} d \delta\left(\partial_{\theta} A_{\delta}-\partial_{\delta} A_{\theta}\right)
$$

where the Berry connection $A_{\mu} \equiv i\left\langle\Psi(\theta, \delta)\left|\partial_{\mu}\right| \Psi(\theta, \delta)\right\rangle$ $(\mu=\delta, \theta)$. We numerically calculate the Chern number $C_{g}$ using the method for a discrete manifold [20]. When the parameter $\alpha=1 / 3$, we find for this boson system that the Chern number $C_{g}=1(-1)$ for the filling fraction $\nu=1 / 3(2 / 3)$ and $C_{g}=0$ when $\nu=1$. As an example, we show the value of $C_{g}$ as a function of $U$ at $\nu=1 / 3$ in Fig. 1, where the manifold of torus is discretized by $5 \times 5$ meshes in the calculation. When the system is in the gapped Mott state with $U>U_{c}, C_{g}$ is quantized to be exactly at 1 , while $C_{g}$ is unquantized when the system enters the gapless superfluid phase. Because of quantization of $C_{g}$, the finite size effect seems to have a minimal influence, and we can use exact diagonalization of a small system to get the exact value of $C_{g}$ in Fig. 1 for the Mott phase (however, because of the finite-size gap, $C_{g}$ is still approximately unity in some region of the superfluid phase near the transition point). This calculation unambiguously shows that this bosonic system is in a topological Mott insulator phase with nonzero Chern number at the fractional filling of the optical lattice.

Edge states.- The appearance of edge states at the boundary is usually considered to be a hallmark of nontrivial topological properties for the bulk system. Under the periodic boundary condition, this interacting system is gapped at the fractional filling $\nu=1 / 3$ (or $2 / 3$ ). However, under the open boundary condition, edge states confined to the boundary can appear inside the energy gap, signaling the nontrivial topological properties of the bulk insulator. The quasiparticle energy spectrum $\Delta E_{n}$ is determined by the additional energy required to add an atom to a system with $n$ atoms, that is,

$$
\Delta E_{n}^{(O, P)} \equiv E_{n+1}^{(O, P)}-E_{n}^{(O, P)},
$$

where $E_{n}^{(O)}\left(E_{n}^{(P)}\right)$ is the ground-state energy of the system with $n$ atoms under the open (periodic) boundary condition [21]. In Fig. 2(a), we show the quasi-particle 
energy spectrum for a system with 96 lattice sites near the filling $\nu=1 / 3$ under both periodic and open boundary conditions. The calculation is done using the density matrix renormalization group method [22], which provides a reliable approach to precisely calculate energies for any $1 \mathrm{D}$ systems. Near the filling $\nu=1 / 3$, the quasiparticle energy spectrum is split into two branches separated by a finite gap. The calculation clearly shows that two states appear in the gap of the energy spectrum under the open boundary condition. In Fig. 2 (b), we show the quasi-particle energy spectrum as a function of phase $\delta$ under the open boundary condition. Inside the gap between the lower and the upper branches of the energy spectrum, one can see two edge modes (which are degenerate in energy at $\delta=2 \pi / 3$ ) that connect these two branches of the bulk spectrum as one varies phase $\delta$.

To verify that the in-gap modes indeed correspond to the edge states, we numerically calculate the excitation distribution of these modes and find that they are confined near the edges of the chain. The distribution of the quasi-particle can be defined as

$$
\Delta n_{j}=\left\langle\Psi_{n+1}^{g}\left|n_{j}\right| \Psi_{n+1}^{g}\right\rangle-\left\langle\Psi_{n}^{g}\left|n_{j}\right| \Psi_{n}^{g}\right\rangle,
$$

where $\left|\Psi_{n}^{g}\right\rangle$ denotes the ground state wave function of the system with $n$ bosonic atoms. The distribution of the ingap quasi-particle modes for $N=96$ sites under filling $\nu=1 / 3$ is plotted in Fig.2 (c). As expected, the in-gap states mainly distribute near the two edges, especially for a large $V$. For instance, $99 \%$ of the quasi-particle modes at $V=10$ are localized at the two edge sites.

Two-component $\mathrm{BH}$ model in a superlattice and spin Chern number.- If the phase $\delta$ in Eq.(2) is replaced by $-\delta$, we find that the Chern number is -1 (1) for $\nu=1 / 3$ $(2 / 3)$, that is, the sign of the Chern number is flipped. This fact implies that we can realize a topological insulator characterized by a nontrivial spin Chern number with a two-component bosonic gas in a 1D optical superlattice. To this end, we consider a simple case where the intercomponent atomic collision is turned off, e.g., through Feshbach resonance, and the system is described by a decoupled two-component $\mathrm{BH}$ model with the Hamiltonian

$$
H=-J \sum_{\langle i j\rangle \sigma} b_{i \sigma}^{\dagger} b_{j, \sigma}+\sum_{j, \sigma}\left[\frac{U_{\sigma}}{2} n_{j \sigma}\left(n_{j \sigma}-1\right)+V_{j \sigma} n_{j \sigma}\right]
$$

where the potential $V_{j \sigma}=V \cos \left(2 \pi \alpha j+\delta_{\sigma}\right)$ with $\delta_{\uparrow}=$ $-\delta_{\downarrow}=\delta, b_{j \sigma}$ denotes the bosonic annihilation operator with the pseudo-spin $\sigma=\uparrow, \downarrow$, and $U_{\sigma}$ is the intracomponent interaction rate for $\operatorname{spin} \sigma$. For this decoupled two-component $\mathrm{BH}$ model, we have the Chern number $C_{g}^{\uparrow}=-C_{g}^{\downarrow}$. So, although the total charge Chern number $C_{g}^{\uparrow}+C_{g}^{\downarrow}$ cancels out to zero, the spin Chern number $C_{g}^{s}=C_{g}^{\uparrow}-C_{g}^{\downarrow}$ [23] is non-vanishing at fractional fillings. The nonzero spin Chern number is usually associated with the quantum spin Hall effects in two-dimensional
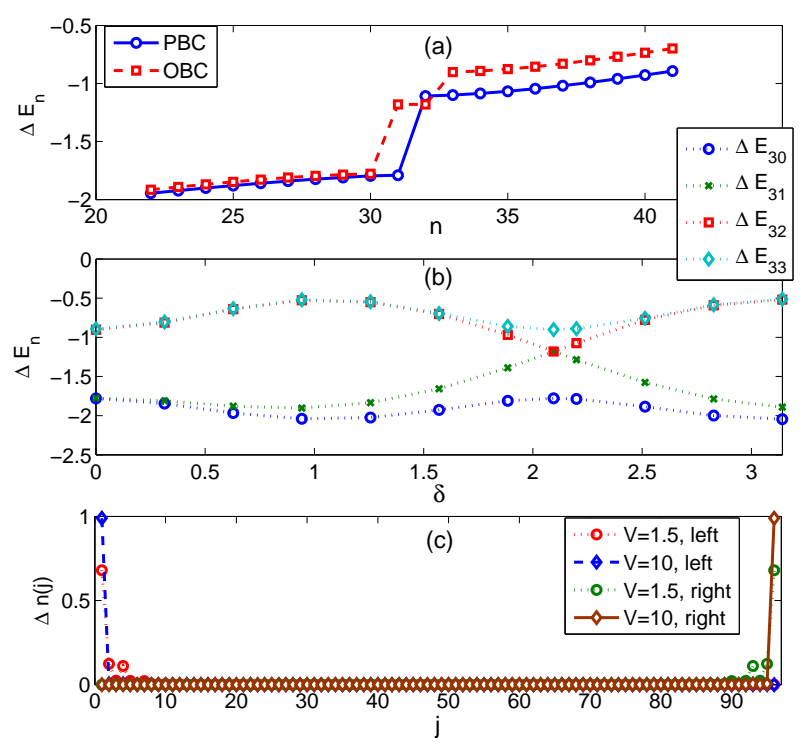

FIG. 2: (Color online) (a) The quasi-particle energy spectrum $\Delta E_{n}$ [see the definition by Eq. (3)] versus $n$ under the periodic $(\mathrm{PBC})$ or open $(\mathrm{OBC})$ boundary condition. The calculation is done in a 96-site lattice near the filling $\nu=1 / 3$ with $V=1.5, U=10, \delta=2 \pi / 3$, and $\alpha=1 / 3$. (b) The edges of the lower $\left(\Delta E_{30}\right)$ and the upper $\left(\Delta E_{33}\right)$ branches of the energy spectrum and the two in-gap modes $\left(\Delta E_{31}\right.$ and $\left.\Delta E_{32}\right)$ as functions of the phase $\delta$ under the open boundary condition. (c) The distribution of the two in-gap modes along the chain at $V=1.5,10$. The other parameters for (b) and (c) are the same as those for (a).

systems [23]. For our 1D system, spin edge states appear when the spin Chern number is nonzero. For an example with $\alpha=1 / 3$, we have $C_{g}^{s}=2$ at the fractional fillings $\nu=1 / 3(2 / 3)$ and $C_{g}^{s}=0$ at the integer filling. The edge states are similar to those shown in Fig. 2. The spin up (down) edge state is confined near the left (right) edge, respectively.

Experimental detection.- We now discuss how to measure topological Chern number in a practical experimental setting. For atomic experiments, apart from the optical superlattice potential, the bosons are confined in a weak global harmonic trap. For simplicity, we consider the large- $U$ limit where the system is described by hard-core bosons with no more than one atom occupying the same lattice site. The total potential, including the optical superlattice and the global harmonic trap, is described by

$$
V_{j}=V \cos (2 \pi \alpha j+\delta)+V_{H}\left(j-j_{0}\right)^{2},
$$

where $j_{0}$ denotes the position of the trap center and $V_{H}$ is the strength of the harmonic trap. We use the Jordan-Wigner transformation, $b_{j}^{\dagger}=f_{j}^{\dagger} \prod_{m=1}^{j-1} e^{-i \pi f_{m}^{\dagger} f_{m}}$ and $b_{j}=\prod_{m=1}^{j-1} e^{i \pi f_{m}^{\dagger} f_{m}} f_{j}$, to map the hard-core $\mathrm{BH}$ 
model to non-interacting fermion Hamiltonian $H_{F}=$ $-J \sum_{j}\left(f_{j}^{\dagger} f_{j+1}+\right.$ h.c. $)+\sum_{j} V_{j} f_{j}^{\dagger} f_{j}$, where $f_{j}^{\dagger}$ and $f_{j}$ are the creation and annihilation operators for spinless fermions, respectively [17, 24]. The particle density of hard-core bosons coincides with that of non-interacting fermions as we have $n_{j}=\left\langle b_{j}^{\dagger} b_{j}\right\rangle=\left\langle f_{j}^{\dagger} f_{j}\right\rangle=n_{j}^{F}$ with the Jordan-Wigner transformation; however, the momentum distribution for bosons is typically very different from that for fermions.

After the hard-core bosons are mapped to fermions, there is a simple way to figure out the Chern number. The ground state of free fermions is a Slater determinant, i.e., a product of single particle states $\left|\Psi_{g}^{F}\right\rangle=$ $\prod_{m=1}^{N_{f}} \sum_{n=1}^{N} P_{n m} f_{n}^{\dagger}|0\rangle$, with $N_{f}=N_{b}$ the number of fermions and $P$ the matrix of the components of $\left|\Psi_{g}^{F}\right\rangle$. Supposing that the $n$-th eigenstate of a single particle is denoted by $\left|\psi_{n}\right\rangle=\sum_{j} \phi_{j, n} f_{j}^{\dagger}|0\rangle$, the eigenvalue equation $H_{F}\left|\psi_{n}\right\rangle=E_{n}\left|\psi_{n}\right\rangle$ can be written in terms of the following Harper equation 11]

$$
-J\left(\phi_{j+1, n}+\phi_{j, n}\right)+V \cos (2 \pi \alpha+\delta) \phi_{j, n}=E_{n} \phi_{j, n},
$$

where $\phi_{j, n}$ is the amplitude of the particle wave function of the $j$-th site and $E_{n}$ is the $n$-th single-particle eigen-energy. Compared with the Harper equation in a magnetic field, we know that $\alpha$ corresponds to the magnetic flux [14]. Therefore, we can define the local density difference as

$$
\delta n_{j}=\frac{n_{j}\left(\alpha_{1}\right)-n_{j}\left(\alpha_{2}\right)}{\alpha_{1}-\alpha_{2}} .
$$

The Chern number $C_{g}$ can then be obtained through the Streda formula $C_{g}=\delta n_{j}$ under the condition that $n_{j}\left(\alpha_{\eta}\right)$ $(\eta=1,2)$ is the local density associated with the plateau at $\alpha_{\eta}[7,8,11,12]$.

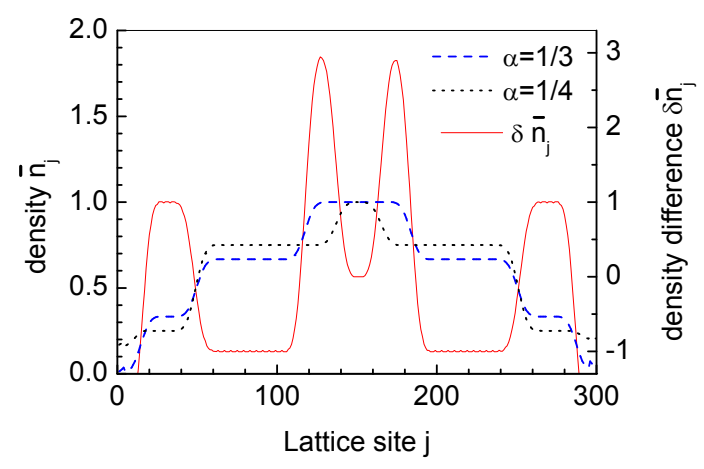

FIG. 3: (Color online) The average density profiles $\bar{n}_{j}$ and the density difference $\delta \bar{n}_{j}$ for $\alpha_{1}=1 / 3$ and $\alpha_{2}=1 / 4$. The values of $\delta \bar{n}_{j}$ represent the corresponding Chern numbers at the plateaus with the fillings $\nu=1 / 3,2 / 3,1$ for $\alpha=1 / 3$ and $\nu=1 / 4,3 / 4,1$ for $\alpha=1 / 4$. The other parameters are $N=$ $300, N_{b}=180, M=4, V=10, \delta=\pi / 2$, and $V_{H}=0.001$.

Following the method outlined in Ref. [17], we numerically calculated the average density profiles for $\alpha=$
$1 / 3,1 / 4$, with the results shown in Fig.3. To reduce the oscillations in density profiles induced by modulation of the potentials, we define the local average density $\bar{n}_{j}=\sum_{m=-M}^{M} n_{j+m} /(2 M+1)$, where $2 M+1$ is the length to average the density, which corresponds to the position resolution in the experimental detection. We take $M \ll N$, e.g., $M=4$ and $N=300$ in Fig. 3, as it is typical for experiments. As one can see from the density profiles $\bar{n}_{j}$ in Fig. 3, plateaus appear at the rational fillings $\nu=1 / 3,2 / 3,1$ for $\alpha=1 / 3$, and $\nu=1 / 4,3 / 4,1$ for $\alpha=1 / 4$ (the gap at half filling in the case of $\alpha=p / q$ with an even $q$ is generally closed at an integer $\delta / \pi[14])$. Using the Streda formula [Eq. (8)], we obtain $C_{g}=\delta n_{j}=1,-1$ at the fractional fillings $\nu=\alpha, 1-\alpha$, and $C_{g}=0$ at the integer filling $\nu=1$. The width of the plateaus is associated with the size of the energy gap. To make detection of the Chern number easier, we can adjust the frequency of the harmonic trap to move the target plateaus to the center of the trap. For example, if we choose $V_{H}=10^{-4} J$ and other parameters as those given in Fig.3, the plateaus at $\nu=2 / 3$ for $\alpha=1 / 3$ and $\nu=3 / 4$ for $\alpha=1 / 4$ are moved to the center of the trap spanning from the 65 th to the 235th lattice site. With such a wide plateau, it is straightforward to read out the Chern number $C_{g}=\delta \bar{n}_{j}=-1$ for this case.

In summary, we have shown that for bosonic atoms in a 1D optical super-lattice, the Mott insulator states of the corresponding $\mathrm{BH}$ model at fractional fillings are topologically nontrivial, characterized by nonzero Chern number and existence of edge states. We further predict that the topological Chern number can be detected by measuring the plateaus in the density profile when the atoms are trapped in a global harmonic potential. The model discussed in this Letter represents one of the simplest experimental systems to show intriguing topological properties, and the proposed detection method allows one to confirm these topological properties with the state-of-the-art technology.

SLZ is supported by the NSF of China (Grant No. 11125417), the SKPBR of China (Grant No.2011CB922104), and the PCSIRT. ZDW is supported by the GRF (HKU7058/11P) and the CRF (HKU8/11G) of Hong Kong RGC. LMD and YHC acknowledge support by the NBRPC (Grant No.2011CBA00302), the DARPA OLE Program under ARO Award W911NF0710576, the IARPA, and the AFOSR and ARO MURI program.

[1] M. Lewenstein, A. Sanpera, V. Ahufinger, B. Damski, A. Sen(De), and U. Sen, Adv. Phys. 56, 243 (2007); I. Bloch, J. Dalibard, and W. Zwerger, Rev. Mod. Phys. 80, 885 (2008).

[2] D. Jaksch, C. Bruder, J. I. Cirac, C. W. Gardiner, and P. Zoller, Phys. Rev. Lett. 81, 3108 (1998). 
[3] M. Greiner, O. Mandel, T. Esslinger, T.W. Hansch, and I. Bloch, Nature (London) 415, 39 (2002); T. Stoferle, H. Moritz, C. Schori, M. Kohl, and T. Esslinger, Phys. Rev. Lett. 92, 130403 (2004).

[4] L.-M. Duan, E. Demler, and M. D. Lukin, Phys. Rev. Lett. 91, 090402 (2003).

[5] M.Z.Hasan and C. L.Kane, Rev. Mod. Phys. 82, 3045 (2010); X. L. Qi and S. C. Zhang, Rev. Mod. Phys. 83, 1057 (2011).

[6] S. L. Zhu, H. Fu, C. J. Wu, S. C. Zhang, and L. M. Duan, Phys. Rev. Lett. 97, 240401 (2006).

[7] R. O. Umucalilar, H. Zhai, and M. O. Oktel, Phys. Rev. Lett. 100, 070402 (2008).

[8] L. B. Shao, S. L. Zhu, L. Sheng, D. Y. Xing, and Z. D. Wang, Phys. Rev. Lett. 101, 246810 (2008).

[9] N. Goldman, I. Satija, P. Nikolic, A. Bermudez, M. A. Martin-Delgado, M. Lewenstein, and I. B. Spielman, Phys. Rev. Lett. 105, 255302 (2010).

[10] E. Alba, X. Fernandez-Gonzalvo, J. Mur-Petit, J. K. Pachos, and J. J. Garcia-Ripoll Phys. Rev. Lett. 107, 235301 (2011)

[11] L. J. Lang, X. Cai, and S. Chen, Phys. Rev. Lett. 108, 220401 (2012).

[12] F. Mei, S. L. Zhu, Z. M. Zhang, C. H. Oh, and N. Goldman, Phys. Rev. A 85, 013638 (2012).

[13] D. J. Thouless, M. Kohmoto, M. P. Nightingale, and M. den Nijs, Phys. Rev. Lett. 49, 405 (1982).

[14] A. Bohm, A. Mostafazadeh, H. Koizumi, Q. Niu, and J. Zwanziger, The Geometric Phase in Quantum Systems,
( Spinger-Verlag, Berlin,2003).

[15] S. Raghu, X. L. Qi, C. Honerkamp, and S. C. Zhang, Phys. Rev. Lett. 100, 156401 (2008).

[16] G. Roati, C. D'Errico, L. Fallani, M. Fattori, C. Fort, M. Zaccanti, G. Modugno, M. Modugno, and M. Inguscio, Nature (London) 453, 895 (2008); L. Fallani, J. E. Lye, V. Guarrera, C. Fort, and M. Inguscio, Phys. Rev. Lett. 98, 130404 (2007).

[17] V. G. Rousseau, D. P. Arovas, M. Rigol, F. Hebert, G. G. Batrouni, and R. T. Scalettar, Phys. Rev. B 73, 174516 (2006).

[18] For a pedagogical introduction to the exact diagonalization of the BH model, see M. Zhang and R. X. Dong, Eur. J. Phys. 31, 591 (2010).

[19] Q. Niu, D. J. Thouless, and Y. S. Wu, Phys. Rev. B 31, 3372 (1985).

[20] T. Fukui, Y. Hatsugai, and H. Suzuki, J. Phys. Soc. Jpn. 74, 1674 (2005).

[21] H. Guo, S. Q. Shen, and S. Feng, Phys. Rev. B 86, 085124 (2012); H. Guo and S. Q. Shen, Phys. Rev. B 84, 195107 (2011).

[22] U. Schollwöck, Rev. Mod. Phys. 77, 259 (2005).

[23] D. N. Sheng, Z. Y. Weng, L. Sheng, and F. D. M. Haldane, Phys. Rev. Lett. 97, 036808 (2006).

[24] B. Paredes, A. Widera, V. Murg, O. Mandel, S. Folling, I. Cirac, G. V. Shlyapnikov, W. Hansch, and I. Bloch, Nature (London) 429, 277 (2004). 